\title{
Biomedical Image Compression Techniques for Clinical Image Processing
}

\author{
https://doi.org/10.3991/ijoe.v16i12.17019
}

\author{
Abdul Khader Jilani Saudagar \\ Imam Mohammad Ibn Saud Islamic University (IMSIU), Riyadh, Saudi Arabia \\ aksaudagar@imamu.edu.sa
}

\begin{abstract}
Image processing is widely used in the domain of biomedical engineering especially for compression of clinical images. Clinical diagnosis receives high importance which involves handling patient's data more accurately and wisely when treating patients remotely. Many researchers proposed different methods for compression of medical images using Artificial Intelligence techniques. Developing efficient automated systems for compression of medical images in telemedicine is the focal point in this paper. Three major approaches were proposed here for medical image compression. They are image compression using neural network, fuzzy logic and neuro-fuzzy logic to preserve higher spectral representation to maintain finer edge information's, and relational coding for inter band coefficients to achieve high compressions. The developed image coding model is evaluated over various quality factors. From the simulation results it is observed that the proposed image coding system can achieve efficient compression performance compared with existing block coding and JPEG coding approaches, even under resource constraint environments.
\end{abstract}

Keywords-Accuracy, fuzzy logic; image compression; neural networks, telemedicine.

\section{$1 \quad$ Introduction}

Telemedicine is one of the poorest areas of the main attractions, particularly those remotely located from major population centers is capable of providing specialist medical care. Telemedicine has the potential to improve the accessibility of specialist medical care to people in remote areas, and in turn help to fight preventable diseases. It also cost and regional centers will have a huge impact on the necessity of transporting patients. A primary means of identifying patients who require emergency treatment but also that Tele-medicine has a role. In this manner, the cost of expenditure in sending the medical teams to isolate, remote and sparse areas can be reduced.

The important equipment of a telemedicine system "see Figure 1" with store and forward capability includes a data recording system with good quality, an efficient communication system operating by a well-trained person, data viewing system which can be accessible by a specialist in medical processing and data archiving equipment [1]. In the telemedicine-oriented applications, where visual imaging plays a vital role 
in the medical diagnosis, the images with good quality (for example retina and an external eye) form the most important parts of the system.

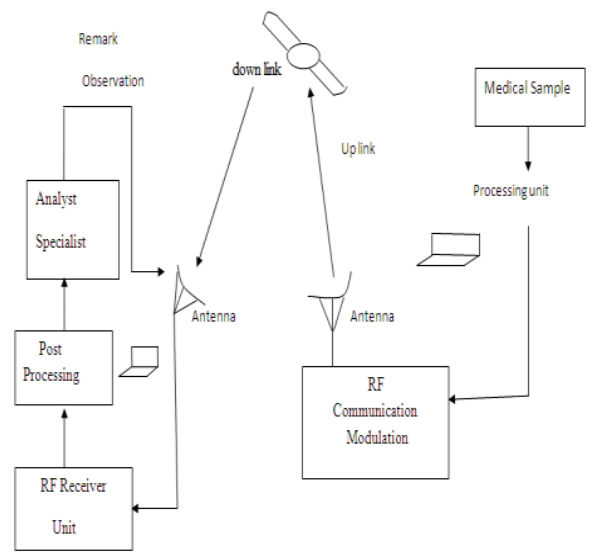

Fig. 1. The telemedicine approach for remote telemedicine application

Though the technical challenges such as image capturing, digitization and encoding are addressed easily, the two issues are linked to the telemedicine system that can make the entire system ineffective. The communication system will exist in some areas and not exists in some areas. In the cases where there is no existence of proper communication system, satellite phones can be utilized but the system achieves low data rate both in transmitting and receiving and also this equipment is very expensive. In addition, the digitized visual images consume more storage space and also take more time to transmit. For instance, a visual image with high quality may achieve a size of 1.5 MB or greater. Over a system with a baud rate of 9600 bauds/sec, the entire image transmission will consume an approximately 25 minutes. This will become critical if there is large number of images to transmit. Figure 2 shows the effect of encoding of a long range medical retinal image processing.

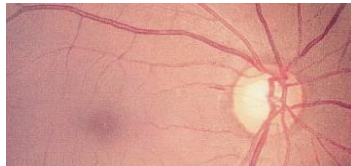

(a)

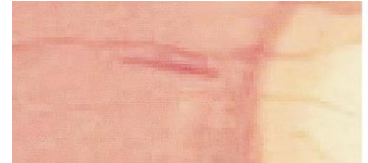

(b)

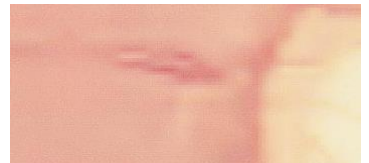

(c)

Fig. 2. (a-c) Retina image with a nerve fiber layer close to the optic disc

To process this type images, a compression unit is required and is explained in the following paragraphs. In telematics applications, especially in telemedicine [2], image compression has significance due to its advantages of storage reduction and optimized resource utilization [3]. Sometimes it is required to transmit a single image or multiple images over far distances through the computer networks which will be useful for 
multipurpose. For example, for a fast, reliable and improved diagnosis at various centers, the transmission of multiple medical images captured at one place is going to take place. Thus, to achieve fast, reliable and an improved diagnosis, the image compression should be in an efficient way.

However, the main problem in image compression, if there is a requirement of high compression rates, the applicability of important characteristics of medical image are preserved or not is needs to be verified over the reconstructed medical image. The medical applications [4], diagnosis will be effective if the compression approach is able to preserve all the important and relevant features of image. This is the main drawback of lossless compression approaches [5]. In the view of encoding and storage, the lossy compression has better performance but there is no warranty about the preservation of important features which are very useful for accurate diagnosis in medical image processing applications. In the lossy compression, the characteristics are preserved in the space domain in which the original image is getting transformed. i.e., in the case of image compression with DWT, the entire information which is required to medical image reconstruction will be stored in the wavelet coefficients.

\subsection{Compression model}

The most common image compression model in the encoding of images is JPEG (Joint Photographic Experts Group) compression, can also be used for medical image compression in medical image processing applications. In image compression, to obtain an efficient compression rate, there will be a minimum degradation in the quality of image and also will be some information loss. Different types of coding approaches were developed to process medical images. The two basic approaches used are:

- Predictive coding and

- Transform coding

Predictive coding is a coding technique in which the information which is already available or sent is used to estimate the future values and then difference between the original and estimated values is coded. Predictive coding is accomplished on the pixel of image in image or spatial domain, it can be adapted to image properties readily and also it is very simple to implement. Differential Pulse Code Modulation (DPCM) [6] is one of the examples for predictive coding.

On the other hand, the transform coding initially transform the original image present in the spatial domain into some other domain using some well-known transform and then encodes the transformed coefficients. Transform coding has greater complexity [7] when compared it with predictive coding but also provides an efficient compression rates. The Block diagram of Transform-coding is as represented in the Figure 3. 


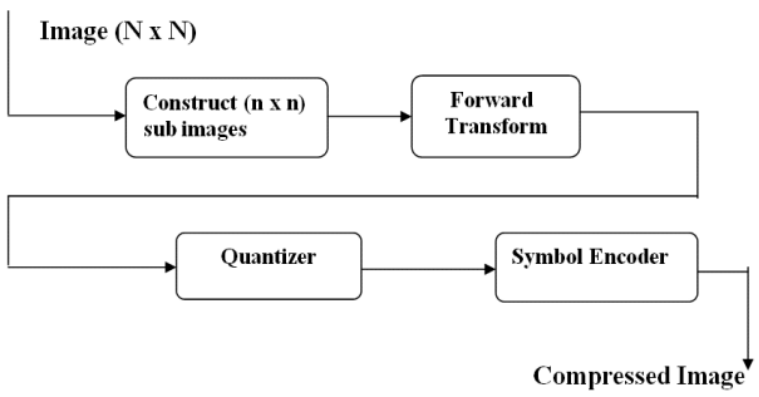

Fig. 3. Block diagram of transform coding

It is observed that this approach results in a huge coefficient count and quantization error, demands in higher resources and lower compression rate. These drawbacks made the conventional image coding techniques less applicable to real time scenarios. The integrated model of neural network and fuzzy logic is defined for the image compression application. To validate the proposed approach, the suggested method is tested over different medical samples and the obtained results are compared with the conventional JPEG based coding approach.

The developed work is completely represented in eight sections. Section 1 illustrates the introduction of the developed work. Section 2 illustrates the details of earlier developed medical image compression approaches. Various suggestions given by various authors for the focused problem discussed in this section. The compression method through various coding techniques and the methodology accomplished to perform such coding is outlined. Section 3 outlines the proposed hybrid image coding model. This hybrid coding is developed by combining fuzzy logic and neural network. Various image samples are evaluated to check the performance of proposed system and the obtained results are described in this section 4. A comparative analysis is also illustrated in this section through PSNR and computation time. The conclusions and future scope of the developed work is outlined in section 5 .

\section{$2 \quad$ Literature Review}

Automated image compression systems can be developed using Artificial Neural Networks (ANN) and Fuzzy Logic individually and when used together gives superior results. The ANN is an information processing standard, developed with the base of the working principle of biological nervous system, such as brain and its processing of information through nerves. The important component of this ANN system is the novel structure of data processing system. It is constructed with a lot of interconnected processing elements, called as neurons, working with a united fashion to solve the particular problems. An ANN is designed for particular application, such as classification of data, pattern recognition, through a process of learning. Learning will be adjustments to the synaptic connections between neurons that exist in biological systems. 
Many image compression techniques using neural networks were proposed in the past. One such image compression technique proposed in [8] to balance the tradeoff between image quality and encoding speed. This approach combined the bipolar interpolation with NN. Here the bipolar interpolation describes the interpolation of primitive and non-primitive regions of the image. From the obtained results it was observed that this approach is superior to all conventional approaches in the view of visual quality of the reconstructed image, smoothness in the edge regions of the interpolated images and the clarity. A similar NN based compression is proposed by Yongxue and Hui [9] based on the wavelet packet transform. This approach used haar wavelet as a wavelet filter and the image was reconstructed by wavelet packet at receiver side. The main fact behind this approach is the orientations of wavelet packets are often similar and can be encoded through the same code words through the "Vector Quantization" algorithm.

Mustafa \& Abdullah [10] introduced neural network approach with arithmetic coding. The coding is by using perceptron neural network to compress each three-pixel into single value; this value is called Compression value. In the decompression part we use Genetic Algorithm for return the pixels, by using Crossover operation and the Fitness Value and results in satisfactory performance in terms of compression ratio and quality. This method is tested with color images in medical processing applications. The similar concept is better explained in [11]. Here the successful image compression and image decompression is done by the "counter propagation NN". The CPNN also had shown the robustness towards various types of medical images.

An extension to "BS-CROI" is developed in [12] to attain better retrieval quality. In this approach, the design of $\mathrm{NN}$ is of network cascading with one node of hidden layer. Grey level image redistribution is accomplished in the training phase in a random manner to obtain very less "Mean Square Error (MSE)" such that it is applicable for various types of images. The proposed cascaded $\mathrm{NN}$ is observed to be superior compared with conventional fixed NN architecture particularly at high compression ratios. From the above analysis it can be observed that the image compression with AI using NN can achieve effective performance compared to the conventional image compression approaches. Thus, this work focused on the design of an effective medical image compression based on Neural Network.

A new medical image coding is developed in this work based on the neural network and modified coefficient coding. Medical image processing algorithms are generally sensitive to noise and consist of low informative regions. A modified approach to coefficient coding using neural network, for coefficient selection is proposed for the removal of non-informative pixel in medical image coding. Wherein in conventional Filtration the vectors [13], [14] are ranked by some criteria and the top-ranking point is used to the replace the center point. No consideration is made to determine if that center point is original data or not. The unfortunate drawback of using these filters [15], [16], [17], [18] is the smoothing that occurs uniformly across the image. Across areas where there is no noise, original medical image data is removed unnecessarily. In the proposed neural approach, a spatial correlation between each point within the block is computed, and an attempt is made to use this information to first 
decide if the block's center point is a non-informative pixel. If the determination is made that a point is non-informative, the point will be discarded from coding.

In the coding approach to image compression, pixels are selected for compression based on content relevancy. New coding approach was defined to extract the significant pixel coefficient based on pixel relevancy. Towards developing a proper coefficient selection, a fuzzy based image compression approach is developed, carried out over the DCT based image coding. A new image compression method based on fuzzy logic [19] and discrete cosine transform was introduced for medical images together with pre- filter and image enhancing based on fuzzy logic. This method was applied over different sample images and high compression ratios and good validity measures were observed.

A new image compression approach is proposed by Zaheruddin and Mishra [7] by integrating the Fuzzy logic with NN. This approach works based on the concept of learning or determining the relations between input and output images based on their numerical relations. A similar approach was developed in [12], [20] in which a modified pre-processing algorithm was included in the NN. The complete method was carried out in two phases. The first phase presents "BS-CROI" approach to select the image and image compression through back propagation, different to ROI. The obtained PSNR and MSE declared that the proposed approach outperforms the conventional approaches.

Further, the suguno model is incorporated through a multi-level fuzzy rule for medical image sample compression. The basic objective of incorporation of multi-level fuzzy logic is to acquire much finer information for preserving the edge information. The Multiple rule definition results in lowering the coefficient number than the conventional JPEG image coding approach, by a hierarchical coding approach developing the inter band relationship among the pixel. This fuzzy set is then integrated with the learning approach of neural network model to achieve higher level of image compression in medical imaging application.

Researchers in the past suggested many approaches for image compression [21], [22], [23] especially for medical image compression in telemedicine applications using Neural Networks [24], [25], [26], [27], [28], [29], [30], [31] and few works suggested using fuzzy logic [32], [33]. All these suggested approaches have their own advantages and disadvantages.

\section{$3 \quad$ Research Methodology}

This section outlines an effective image compression approach based on Neurofuzzy model which combines the advantages of fuzzy vector quantization with neural network. The emphasis here is on the usefulness of fuzzy vector quantization when it is combined with conventional image coding techniques. The implementation consists of three steps. First, the image is computed for different energy level using DCT transform to obtain a distinct energy representation of the image. Each energy coefficient can be subsequently processed in parallel this will in turn increase the speed to much faster than conventional system. Different quantization and coding schemes are 
used for different energy levels based on their statistical properties. At the second step, coefficients corresponding to lowest energy level are compressed using differential pulse code modulation. Neural network is used to extract the principal components of the higher energy coefficients. Finally, results of the second step are used as input to the fuzzy vector quantization algorithm. The derived simulation results show an encouraging results and superior reconstructed images are achieved. The effect of noise on the compression performance is also studied.

\subsection{Image compression model}

Figure 4 and Figure 5 show the encoding and decoding unit of proposed NeuroFuzzy model.

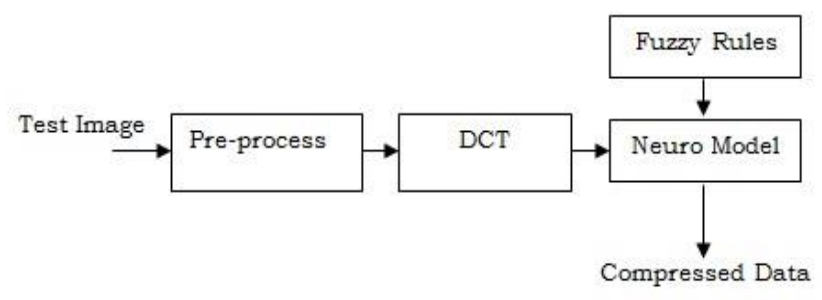

Fig. 4. Encoding Unit for the proposed Neuro-Fuzzy model

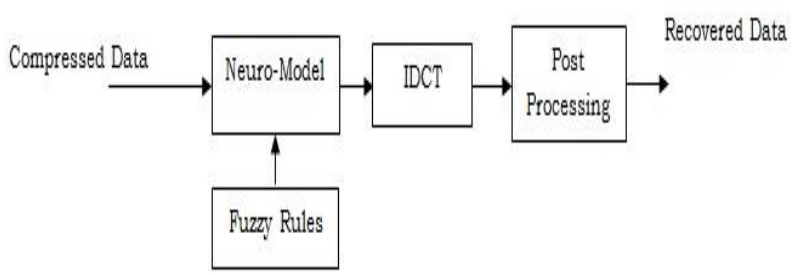

Fig. 5. Decoding unit for the proposed Neuro-fuzzy model

\subsection{Neuro compression model}

Because the human visual system has different sensitivity to different frequency bands, the following strategy is adapted for image encoding. A predetermined compression ratio is used for each energy level in DCT coded data. The lowest energy coefficient, coefficient 1 is encoded with Differential Pulse Code Modulation (DPCM) [34]. These coefficients are scalar quantized. The remaining energy coefficients, level 2 to 6 is coded using neural network. As level 2 and 3 contain the similar energy contents at different resolutions, both the coefficients are encoded using the same neural network with eight units input layer nodes, eight output layer nodes and 6 
hidden layer nodes. Level 5 and6 have the similar energy contents for different spectral level and coded using a larger level network [35]. A multi-layered neural network is shown in Figure 6.

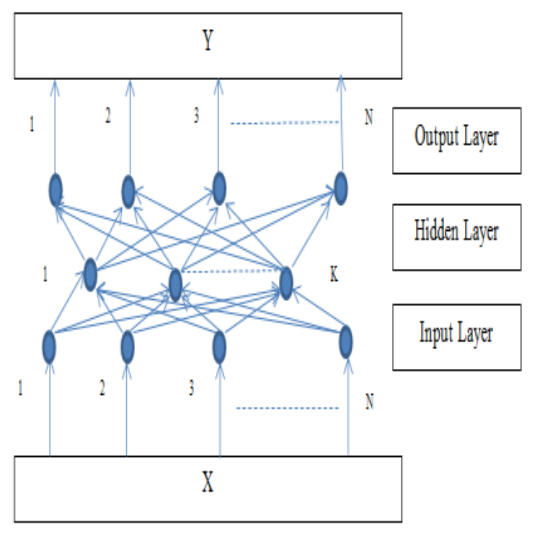

Fig. 6. A multi-layered neural network

Since the energy properties of level 4 coefficients don't match with the energy properties of any other coefficient, they are coded through separate 8-4-8 neural network. Since the level 7 data contributes only a small amount of information, it is removed from the image. From this observation, it can be noticed that this coefficient has only small effect on the reconstructed image due to the zero value. If we denote the weight from $x_{i}$ to $h_{i}$ as $w_{i j}$, then the kelements of hidden vector $\mathrm{h}$ are related to $\mathrm{n}$ elements of input vector $\mathrm{x}$ by equation (1).

$$
h_{i}=\sum_{i=1}^{n} w_{i j} x_{i} j=1,2, \ldots, K
$$

The weight between $h_{i}$ and $y_{i}$ is $w_{i j}$, then the $\mathrm{n}$ elements of hidden vector $\mathrm{h}$ by equation (2).

$$
y_{i}=\sum_{i=1}^{n} w_{i j} x_{j} j=1,2, \ldots, n
$$

Here the weights are chosen in an optimized way such that the measured distortion between output and input vector should be minimum. For a given training set, the Back-Propagation algorithm was used to reduce the MSE between input and output vectors. After the optimization, the normalized DCT coefficients are denoted by $\mathrm{x}$ whose range is in between -1 and 1 . The compressed image obtained through the back propagation neural network is done in two phases, training and encoding.

In the beginning train a single network corresponding to a series of images having similar properties and statistics, and then transmit the output of the hidden layer only for a new presented and compressed image. Given that the network is already trained in advance (off-line), the problems of real-time training and convergence are circumvented. Once the network training is completed, it was ready for test. The network parameters are represented through DCT coefficients one at a time. Simply the input 
block is represented as the output block in the compressed format, obtained through hidden layer nodes of the network. Practically, the outputs obtained at hidden layer nodes are further quantized to achieve efficient compression.

\subsection{Quantization}

The output of the hidden layer of neural network is quantized to achieve further compression. Various vector quantization [36] algorithms like k-means, FVQ1, FVQ2 andFVQ3 were used for code book design in different sets of experiments. Finally, these quantized values are entropy encoded using Huffman encoding.

\subsection{Clustering algorithm}

For clustering k-mean [37] approach is used. The k-means algorithm groups the input vectors into k clusters $C_{j}, \mathrm{j}=1, \ldots, \mathrm{k}$. The main objective of this algorithm is to find the centers (centroids) of clusters for every group. This algorithm evaluates the minimized dissimilarity through the following function defined by equation (3),

$$
D=\frac{1}{N} \sum_{i=1}^{N} d i s_{\min }\left(x_{i}\right)=\frac{1}{N} \sum_{i=1}^{N} \min _{j \in C} \operatorname{dis}\left(x_{i}, c_{j}\right)
$$

Where

$\operatorname{dis}\left(x_{i}, c_{j}\right)=$ distance between ith data point $\left(x_{i}\right)$ and jth centroid $\left(c_{j}\right)$.

$\left(c_{j}\right)=$ centroid of jth cluster.

To simplify the representation, the Euclidean distance is used to evaluate the distance between data points and centroids. A binary membership matric (u) of size kxn is used to represnet the clustered groups. If the distance between particular data point and jth centroid is the minimum distance then that data point is represented with 1 otherwise it is zero, represented as equation (4),

$$
u_{i}\left(x_{i}\right)=\left\{\begin{array}{c}
1 \text { if dis }\left(x_{i}, c_{j}\right)=\text { dis }_{\min }\left(x_{i}\right) \\
0 \text { otherwise }
\end{array}\right.
$$

Where $d i s_{\min }$ is the minimum distance between $x_{i}$ and $c_{j}$. Codebook vectors are evaluated by a distortion measure defined by equation (5)

$$
J=\sum_{j=1}^{k} \sum_{i=1}^{N} u_{i}\left(x_{i}\right)\left\|x_{i}-c_{j}\right\|^{2}
$$

Centroids are computed as the mean of all vectors in group $\mathrm{j}$ :

$$
c_{j}=\frac{\sum_{i=1}^{N} u_{j}\left(x_{i}\right) x_{i}}{\sum_{i=1}^{N} u_{j}\left(x_{i}\right)} \forall j=1,2, \ldots, K
$$

While the k-means algorithm converges to a local minimum, it is not guaranteed to reach the global minimum. In addition, the algorithm is very sensitive to the initial codebook. Furthermore, the algorithm is slow since it requires an exhaustive search through the entire codebook during every iteration process. 


\subsection{Fuzzy algorithm}

These algorithms were developed by evolving a fuzzy codebook design process strategy for the transition from soft to crisp decision and defining conditions for constructing a family of membership functions [38]. In the start of the algorithm, the training vectors are assigned to the codebook vectors that are included in a hemisphere centered at the training vector. The membership function tells the possibility of the training vector belonging to a certain cluster. Thus, membership function is a decreasing function of distance and takes values between 0 and 1 . Let $P_{i}^{(t)}$ be theset of the codebook vectors belonging to the hemispherecentered at the training vector during the tth iteration. Initially during the start of the clustering process, each training vector is assigned to every cluster i.e. $P_{i}^{(C)}=0$, where $\mathrm{c}$ is a finite set containing $\mathrm{k}$ codewords. After the th iteration, the hemisphere located at training vector xi the includes the vectors $c^{j} \in P_{i}^{(t)}$ whose distance from xi is less than equal to the average distance between xi and $c^{j} \in P_{i}^{(t)}$ given by equation (7)

$$
\operatorname{dis}_{\text {avg }}^{(t)}\left(x_{i}\right)=\frac{1}{N\left(P_{i}^{(t)}\right)} \sum_{c j \in P_{i}^{(t)}} \operatorname{dis}\left(x_{i}, c_{j}\right)
$$

Where $N\left(P_{i}^{(t)}\right)$ is the total number of elements in the set $P_{i}^{(t)}$. The set $P_{i}^{(t+1)}$ is formed by equation (8)

$$
P_{i}^{(t+1)}=\left\{c^{j} \in P_{i}^{(t)}: \operatorname{dis}\left(x_{i}, c_{j}\right) \leq \operatorname{dis}{ }_{\text {avg }}^{(t)}\left(x_{i}\right)\right\}
$$

By gradually reducing the radius of the hemisphere, the transition from fuzzy to crisp mode is achieved during the algorithm. When the codebook vector contains a single element, the training vector is assigned to that cluster with the closest center. i.e. if $N\left(P_{i}^{(t)}\right)<2$ training set xi istransferred from fuzzy to crisp mode.The membership value indicates the extent to which a training vector belongs to a particular cluster. A valid membership function must follow the following properties:

- If the hemisphere centered at xi includes a single codebook vector $c_{j}^{*}$, the xi is assigned to $j *$ th cluster.

$$
\begin{aligned}
& u_{j}\left(x_{i}\right)=1 \text { if } \operatorname{dis}\left(x_{i}, c_{j}\right)=\operatorname{dis}\left(x_{i}, c_{j}^{*}\right) \\
& u_{j}\left(x_{i}\right)=0 \text { if } \operatorname{dis}\left(x_{i}, c_{j}\right) \neq \operatorname{dis}\left(x_{i}, c_{j}^{*}\right)
\end{aligned}
$$

- If the number of elements in the set $P_{i}^{(t)}$ is more thanone, then the membership function depends on thedistance between xi and $c^{j} \in P_{i}^{(t)}$, that is $u_{j}\left(x_{i}\right)=$ $f\left(\operatorname{dis}\left(x_{i}, c_{j}\right), c^{j} \in P_{i}^{(t)}\right)$ must satisfy following requirements.

a) $u_{j}\left(x_{i}\right)$ is a decreasing function of $\operatorname{dis}\left(x_{i}, c_{j}\right)$

b) $u_{j}\left(x_{i}\right)$ approaches unity as $\operatorname{dis}\left(x_{i}, c_{j}\right)$ approaches zero.

c) $u_{j}\left(x_{i}\right)$ approaches zero as $\operatorname{dis}\left(x_{i}, c_{j}\right)$ approaches $\operatorname{dismax}\left(x_{i}\right)$ 
d) where $\operatorname{dismax}\left(x_{i}\right)=\max _{c^{j} \in P_{i}^{(t)}} \operatorname{dis}\left(x_{i}, c_{j}\right)$.

Using different membership functions and different methods for codebook calculation, is carried out. The algorithm was developed by constructing a family of membership functions satisfying the conditions proposed [39]. FVQ1 algorithm uses the membership function given by equation (9).

$$
u_{j}\left(x_{i}\right)=f\left(\operatorname{dis}\left(x_{i}, c_{j}\right), \operatorname{dismax}\left(x_{i}\right)=\left(1-\frac{\operatorname{dis}\left(x_{i}, c_{j}\right)}{\operatorname{dismax}\left(x_{i}\right)}\right)^{\mu}\right.
$$

where $\mu$ is a positive integer.

The vector assignment is based on crisp decisions toward the end of the algorithm. This is guaranteed by the minimizing the discrepancy measure $\mathbf{J}$ defined in equation (5) with respect to cj which results in equation (6) for the computation of cj. This selection ensures that the algorithm reduces to crisp k-means algorithm after all the training vectors are transferred from fuzzy to crisp mode. For the quantization of the coefficient, the algorithm defines the membership function defined by equation (10) which satisfies the conditions proposed.

$$
u_{j}\left(x_{i}\right)=\frac{1}{\sum_{i=1}^{k}\left(\frac{\operatorname{dis}\left(x_{i}, c_{j}\right)}{\operatorname{dismax}\left(x_{i}\right)}\right)^{\frac{1}{m-1}}}
$$

The codebook vectors are evaluated by equation (11), resulting from the minimization of $J=j\left(c_{j}, j=1,2, \ldots, k\right.$.

$$
c_{j}=\frac{\sum_{i=1}^{N} u_{j}\left(x_{i}\right)^{m} x_{i}}{\sum_{i=1}^{N} u_{j}\left(x_{i}\right)^{m}} \forall j=1,2, \ldots, k
$$

In this algorithm, toward the end of the algorithm, the training vector assignment is entirely based on crisp decision and $u_{j}\left(x_{i}\right)$ takes the value zero and one, regardless of the value of $\mathrm{m}, u_{j}\left(x_{i}\right)^{m} u_{j}\left(x_{i}\right)$.

The codebook vectors are evaluated using crisp formula (6) which guarantees that the codebook vectors are not affected by the training vectors that are assigned a membership value smaller than unity. i.e. if $\mathrm{m} \approx 1, u_{j}\left(x_{i}\right) \approx 1$ and $u_{j}\left(x_{i}\right)^{m} \approx u_{j}\left(x_{i}\right)$ and for fixed $\mathrm{m},\left(u_{j}\left(x_{i}\right)^{m}-u_{j}\left(x_{i}\right)\right)$ increases as $u_{j}\left(x_{i}\right)$ approaches zero. Another advantage of using equation (6) over equation (11) is that the former is less computationally demanding.

\section{$4 \quad$ Results}

The evaluation results along with the performance are presented for the proposed developed approach in this section. The sample sizes of medical images considered for this experiment are $512 \times 512$ pixels. Matlab software with NN toolbox is used to encode medical images at 0.25 bit per pixel in order to obtain simulation results. Visual comparison results of given medial test image and the retrieved image under nor- 
mal conditions for conventional JPEG coding, NN coding, Fuzzy coding and proposed Neuro-Fuzzy coding are as shown in Figure 7.

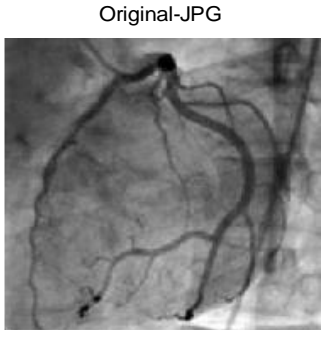

(a)

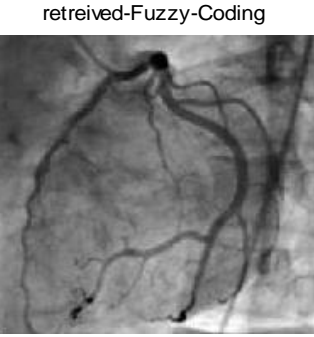

(d)

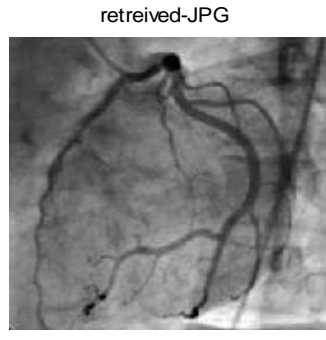

(b)

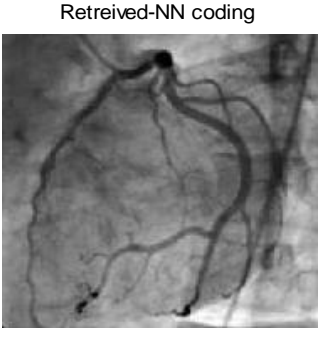

(C)

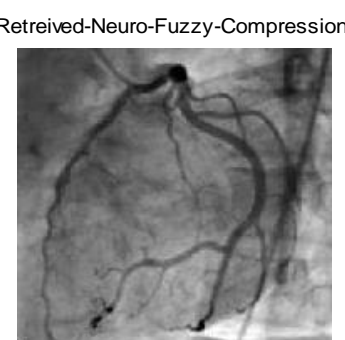

(e)

Fig. 7. (a) Given sample Image, (b) Retrieved sample image using JPEG coding (c) Retrieved sample image using NN coding (d) Retrieved sample image using Fuzzy coding (e) Retrieved sample image using Neuro-Fuzzy coding

The experimental results for the developed Neuro-Fuzzy coding are evaluated using the following parameters and compared with the existing JPEG coding, NN coding and Fuzzy coding.

- Compression Rate (CR)

- Encoding Time (ET)

- Decoding Time (DT)

- Total Time (TT)

- Peak Signal to Noise Ratio (PSNR)

The following figures Figure 8, Figure 9, Figure 10, Figure 11 and Figure 12 shows the intermediary results observed during the execution of Neuro-Fuzzy coding on sample medical image. 


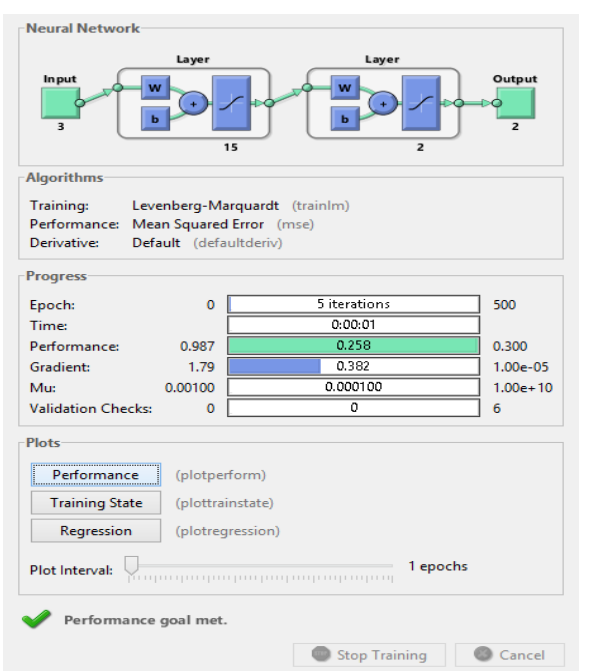

Fig. 8. Neural Network training tool (NN-tool)

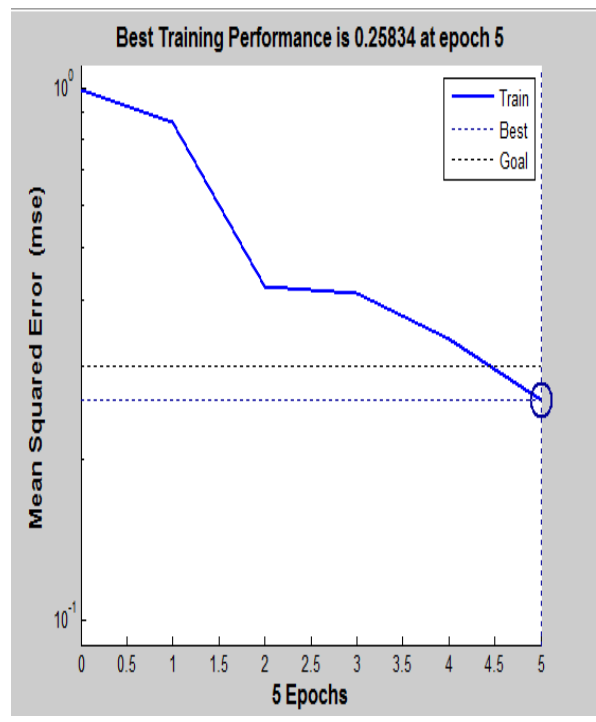

Fig. 9. Best training performance curve 


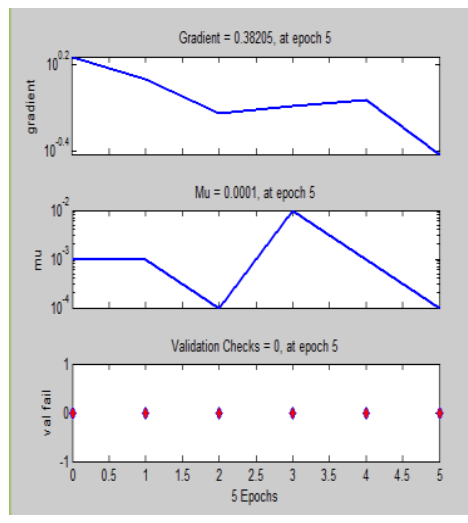

Fig. 10. Training state curve

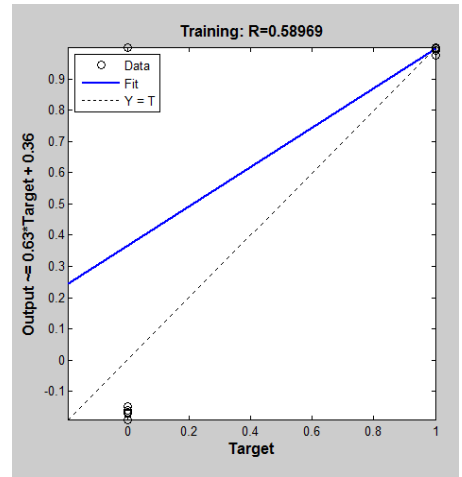

Fig. 11. Regression curve

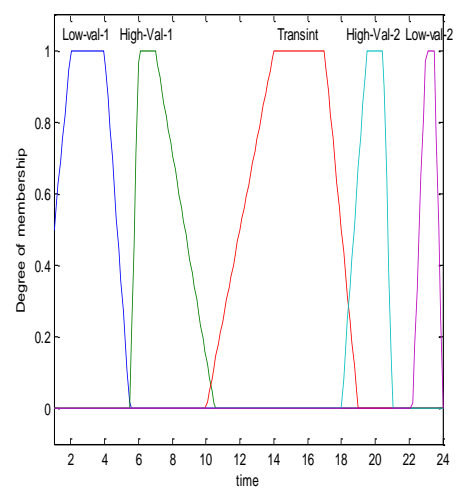

Fig. 12. Degree of membership function for varying time 
Evaluation metric values shown in Table 1 are obtained from the experiment using the above sample medical image depicts the comparison between the developed Neuro-Fuzzy with the earlier JPEG coding, NN coding and Fuzzy coding.

Table 1. Evaluation metrics

\begin{tabular}{|l|c|c|c|c|}
\hline \multicolumn{1}{|c|}{ Metric } & JPEG & NN & Fuzzy & Neuro-Fuzzy \\
\hline Compression Rate (Bpp) & 1.25 & 2.82 & 3.47 & 4.12 \\
\hline Encoding Time Taken $(\mathrm{sec})$ & 5.12 & 6.84 & 5.12 & 10.62 \\
\hline decoding Time Taken $(\mathrm{sec})$ & 9.06 & 2.54 & 2.32 & 2.18 \\
\hline Total processing Time $(\mathrm{sec})$ & 14.18 & 9.39 & 7.45 & 12.81 \\
\hline PSNR (dB) & 40.25 & 50.25 & 50.66 & 53.26 \\
\hline
\end{tabular}

This experiment was performed considering more samples for testing and observed values are noted and presented in Table 2.

Table 2. Evaluation metrics

\begin{tabular}{|l|c|c|c|c|c|c|c|c|c|c|c|c|}
\hline \multirow{2}{*}{ Metric } & \multicolumn{4}{|c|}{ S1 } & \multicolumn{4}{c|}{ S2 } & \multicolumn{4}{c|}{ S3 } \\
\cline { 2 - 15 } & JPEG & NN & Fuzzy & NF & JPEG & NN & Fuzzy & NF & JPEG & NN & Fuzzy & NF \\
\hline CR & 2.015 & 3.493 & 4.3 & 5.10 & 1.203 & 2.762 & 4.075 & 4.03 & 1.671 & 3.253 & 4.00 & 4.75 \\
\hline ET & 5.218 & 6.812 & 4.468 & 7.93 & 4.640 & 7.437 & 4.781 & 8.03 & 5.255 & 6.968 & 4.531 & 7.43 \\
\hline DT & 9.406 & 2.078 & 2.187 & 2.56 & 7.218 & 2.046 & 2.187 & 1.93 & 9.765 & 2.765 & 2.25 & 2.96 \\
\hline TT & 14.62 & 8.890 & 6.653 & 10.0 & 11.85 & 9.484 & 6.968 & 9.96 & 15.01 & 9.734 & 6.781 & 10.4 \\
\hline PSNR & 39.88 & 49.88 & 50.30 & 52.8 & 42.59 & 52.59 & 50.62 & 55.6 & 39.55 & 49.555 & 49.96 & 52.5 \\
\hline
\end{tabular}

The performance obtained for the test medical samples are shown in Figure 13, Figure 14 and Figure 15.

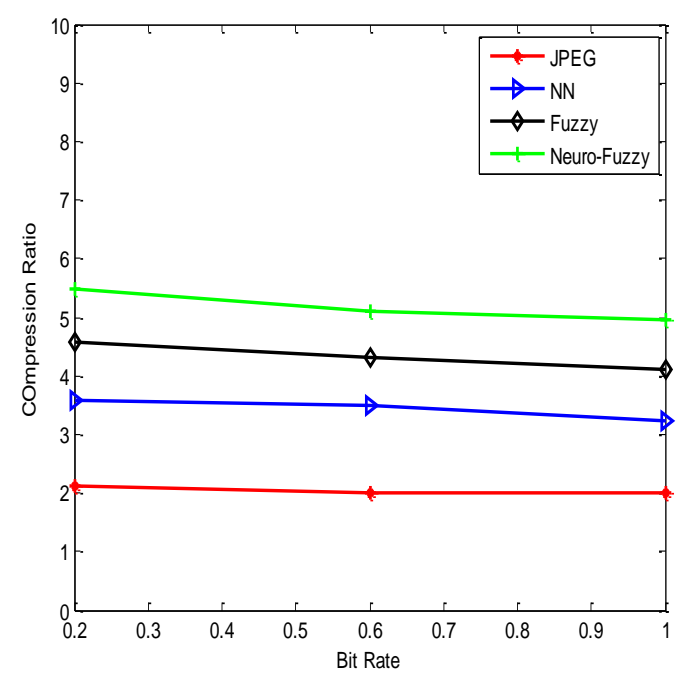

Fig. 13. Comparison of compression rate achieved through JPEG, NN, Fuzzy and NF for the medical test sample 


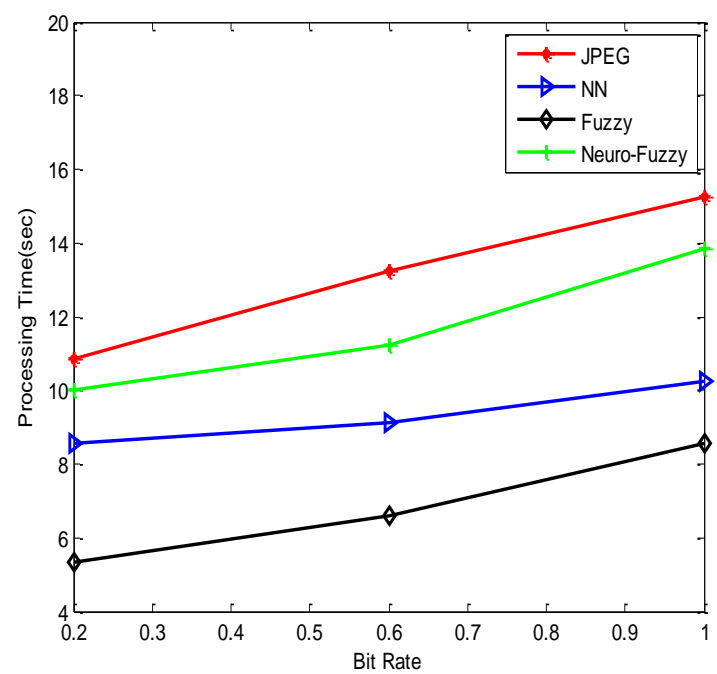

Fig. 14. Comparison of Total Processing Time taken for JPEG, NN, Fuzzy and NF for the medical test sample

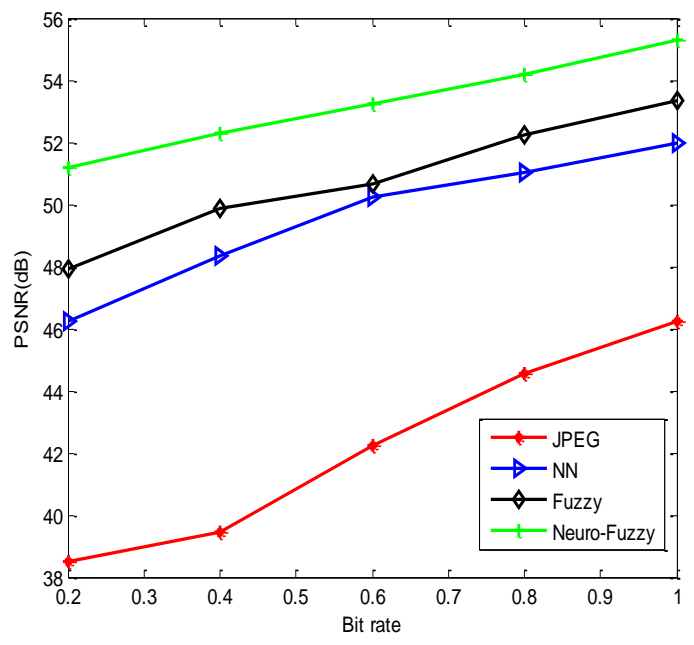

Fig. 15. Comparison of PSNR for JPEG, NN, Fuzzy and NF for varying Bitrate

Figure 16, Figure 17, Figure 18, Figure 19 and Figure 20 shows the comparative analysis of compression rate, encoding time, decoding time, total time and PSNR. 


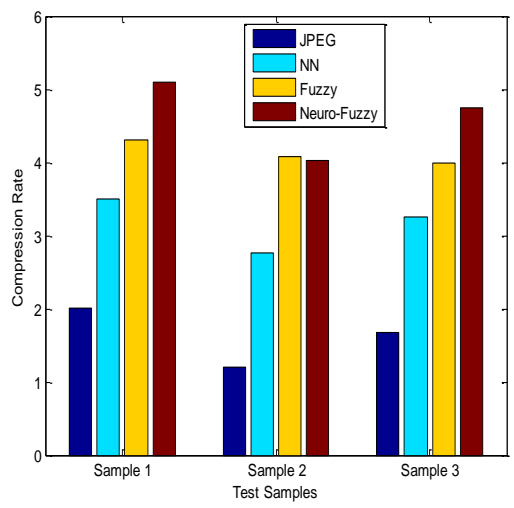

Fig. 16. Comparative analysis of compression rate for various test samples

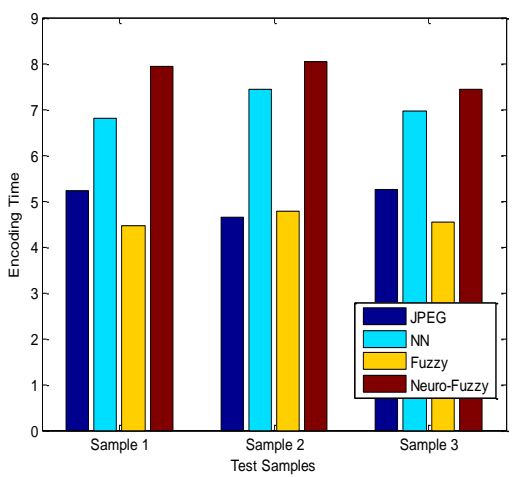

Fig. 17. Comparative analysis of encoding time for various test samples

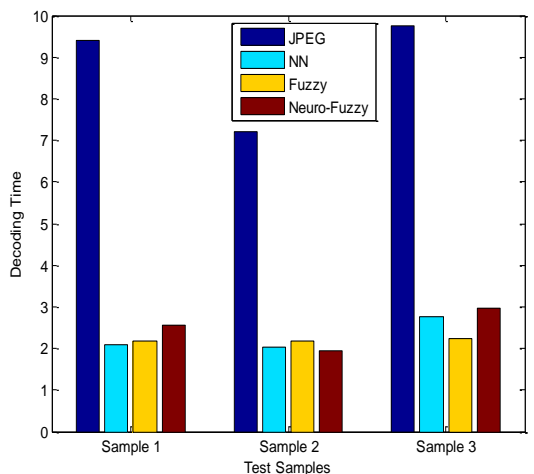

Fig. 18. Comparative analysis of decoding time for various test samples 


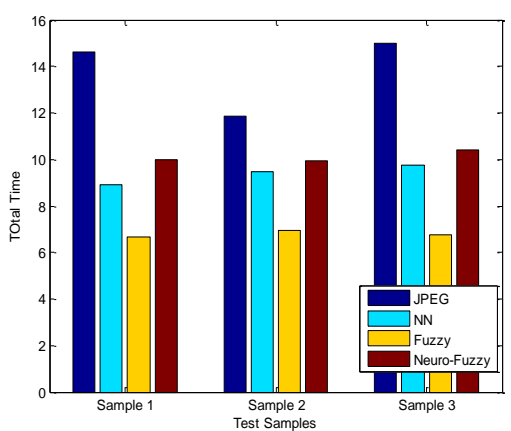

Fig. 19. Comparative analysis total time for various test samples

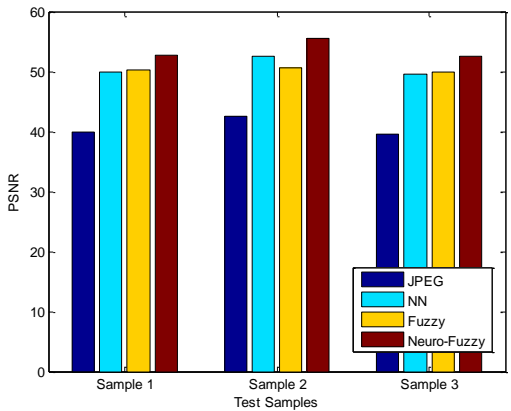

Fig. 20. Comparative analysis of PSNR for various test samples

\section{Conclusion}

The neuro fuzzy model gives a higher performance in image compression as compared to the conventional model of JPEG based coding. The conventional approach has limitations with respect to the bit rate and also the coding methodology. It is also observed that the isolated method of Neural network-based image compression and fuzzy logic-based image compression results in lower accuracy as in comparison to the integrated model of neural network and fuzzy logic. The fuzzy rules and the learning capability of the neural network leads to a proper selection of pixel for coding which result in higher compression ratio with low MSE and higher PSNR value. To evaluate the performance of the proposed system on different test samples (MRI images) and test condition a case analysis to the developed approach can be suggested as future work for this research work. 


\section{$6 \quad$ References}

[1] M. M. Abdellatif and W. Mohamed, "Telemedicine: An IoT based remote healthcare system," International Journal of Online and Biomedical Engineering, vol. 16, no. 6, 2020, pp. 72-81. https://doi.org/10.3991/ijoe.v16i06.13651

[2] B. Karlik, "Medical image compression by using vector quantization neural network (VQNN)," Neural Network World, vol. 16, no. 4, 2006, pp. 341-348. Available: https:// www.researchgate.net/publication/289392327_Medical_image_compression_by_using_Ve ctor_Quantization_Neural_Network_VQNN [Accessed: June 24, 2020]. https://doi.org/10. 1109/glocom.1998.776853

[3] M. B. Martin and A.E. Bell, "New image compression techniques using multiwavelets and multiwavelet packets," IEEE Transactions on image processing, vol. 10, no. 4, April 2001, pp. 500-510. https://doi.org/10.1109/83.913585

[4] A. Said and W. A. Pearlman, "An image multiresolution representation for lossy and lossless compression," IEEE Transactions on Image processing, vol.5, no. 9, September 1996, pp.1303-1310. https://doi.org/10.1109/83.535842

[5] V. D. Raut and S. Dholay, "Analyzing image compression with efficient transforms \& multistage vector quantization using radial basis function neural network," In Proc. IEEE International Conference on Engineering and Technology (ICETECH), 2015, pp. 1-6. https ://doi.org/10.1109/ICETECH.2015.7275009

[6] V. S. Thakur and K. Thakur, "Design and implementation of a highly efficient gray image compression codec using fuzzy based soft hybrid JPEG standard," In Proc. International Conference on Electronic Systems, Signal Processing and Computing Technologies (ICESC), 2014, pp. 484 - 489. https://doi.org/10.1109/ICESC.2014.91

[7] A. Mishra and Zaheeruddin, "Hybrid fuzzy neural network based still image compression", In Proc. International Conference on Computational Intelligence and Communication Networks, 2010. https://doi.org/10.1109/CICN.2010.34

[8] C.T. Lin, K.W. Fan, H.C. Pu, S.M. Lu and S.F. Liang, "An HVS-Directed neural-networkbased image resolution enhancement scheme for image resizing," IEEE Transactions on Fuzzy Systems, vol. 15, no. 4, August 2007, pp. 605-615. https://doi.org/ 10.1109/TFUZZ. 2006.889875

[9] G. Hui and W. Yongxue, "Wavelet packet and neural network basis medical image compression” In Proc. International Conference on E-Product E-Service and E-Entertainment, 2010. https://doi.org/10.1109/ICEEE.2010.5661560

[10] M. S. Mohammed, A. A. Dalya, "Using perceptron neural network and genetic algorithm for image compression and decompression," Journal of Qadisiyah Computer Science and Mathematics, vol. 3, no. 1, 2011, pp. 290-296. Available: http://qu.edu.iq/journalcm/index. php/journalcm/article/view/261 [Accessed: May 22, 2020].

[11] Rekha and V. Saini, "A segmented wavelet inspired neural network approach to compress images," IOSR Journal of Computer Engineering, vol. 2, no. 6, 2012, pp. 36-42. Available: http://www.iosrjournals.org/iosr-jce/papers/vol2-issue6/G0263642.pdf?id=2220 [Accessed: June 15, 2020]. https://doi.org/10.9790/0661-0263642

[12] C. Obiegbu, "Image compression using cascaded neural networks", MS Thesis, University of New Orleans, Louisiana, USA, 2003. Available: https://scholarworks.uno.edu/cgi/view content.cgi? article $=1033 \&$ context=td [Accessed: May 18, 2020].

[13] R. Lukac, “Adaptive vector median filtering," Pattern Recognition Letters, vol. 24, no. 12, 2002, pp. 1889-1899. https://doi.org/10.1016/S0167-8655(03)00016-3 
[14] L. Khriji and M. Gabbouj, "Vector median-rational hybrid filters for multichannel image processing," IEEE Signal Processing Letters, vol. 6, no. 7, 1999, pp. 186-190. https://doi. org/10.1109/97.769365

[15] P. Lambert and L. Macaire, "Filtering and segmentation: the specificity of color images," In Proc. International Conference on Color in Graphics and Image Processing, SaintEtienne, France, September 2000, pp. 57-71.

[16] I. Pitas and P. Tsakalides, "Multivariate Ordering in Color Image Filtering," IEEE Transactions on Circuits and Systems for Video Technology, vol. 1, no. 3, 1991, pp. 247-259. https://doi.org/10.1109/76.97987

[17] R. Lukac, B. Smolka, K. Plataniotis, and A. Venetsanopoulos, "Selection weighted vector directional filters," Computer Vision and Image Understanding, vol. 94, no. 1-3, 2004, pp. 140-167. https://doi.org/10.1016/j.cviu.2003.10.013

[18] M. Vardavoulia, I. Andreadis, and P. Tsalides, "A new median filter for colour image processing,” Pattern Recognition Letters, vol. 22, no. 6-7, 2001, pp. 675-689. https://doi.org/ 10.1016/S0167-8655(00)00141-0

[19] Z. Xiong, K .Ramchandran, and M. Orchad, "Space-Frequency quantization for wavelet image coding," IEEE Transactions Signal Processing, vol. 6, no. 5, May 1997, pp. 677693. https://doi.org/10.1109/83.568925

[20] R. Vijideva, "Neural network- wavelet based dicom image compression and progressive transmission," International Journal of Engineering Science \& Advanced Technology, vol. 2, no. 4, 2012, pp. 1132-1137, 2012. Available: https://www.ijesat.org/volume2issue4.html [Accessed: June 18, 2020].

[21] P. Sreenivasulu and S. Varadarajan, "An efficient lossless ROI image compression using wavelet-based modified region growing algorithm," Journal of Intelligent Systems, vol. 29, no.1, 2020, pp. 1063-1078. https://doi.org/10.1515/jisys-2018-0180

[22] S. N. Kumar, A. L. Fred, H. A. Kumar and P. S. Varghese, "Lossless compression of CT images by an improved prediction scheme using least square algorithm," Circuits, Systems, and Signal Processing, vol 39, 2020, pp. 522-542. https://doi.org/10.1007/s00034-019-01 $\underline{152-8}$

[23] A. S. Mady and S. A. El-Seoud, "An overview of volume rendering techniques for medical imaging," International Journal of Online and Biomedical Engineering, vol. 16, no. 6, 2020, pp. 95-106. https://doi.org/10.3991/ijoe.v16i06.13627

[24] A.K.J. Saudagar, "A case study of evaluation factors for biomedical images using neural networks," In Proc. of the 3rd International Conference on Frontiers of Intelligent Computing: Theory and Applications (FICTA), vol. 327, 2014, pp 241-253. https://doi.org/10.10 07/978-3-319-11933-5 27

[25] A.K.J. Saudagar and A.S. Syed, "Image compression approach with ridgelet transformation using modified neuro modeling for biomedical images," Neural Computing \& Applications, vol. 24, 2014, pp. 1725-1734. https://doi.org/10.1007/s00521-013-1414-y

[26] A.K.J. Saudagar and O. AlShathry, "Neural network-based image compression approach to improve the quality of biomedical image for telemedicine," British Journal of Applied Science \& Technology, vol 4, no.3, 2014, pp. 510-524. https://doi.org/10.9734/BJAST/20 $\underline{14 / 7158}$

[27] A.K.J. Saudagar, "Minimize the percentage of noise in biomedical images using neural networks," The Scientific World Journal, Article ID 757146, 2014. https://doi.org/10.1155/ $\underline{2014 / 757146}$

[28] A. Muharam and A. Ahmad, "Implementation Method On Medical Image Compression System: A Review,” Jurnal Teknologi, vol. 79, no.7, 2017. https://doi.org/10.11113/jt.v79. $\underline{7873}$. 
[29] M. Moorthi and R. Amutha, "High quality model for compression of medical images in telemedicine," Scientific Research and Essays, vol. 8. No. 41, 2013, pp. 2022-2030. https ://doi.org/10.5897/SRE2013.5628

[30] A.K.J. Saudagar, "Learning based coding for medical image compression," International Journal of Advanced Computer Science and Applications, vol.11, no.6, 2020, pp. 111-117. https://doi.org/10.14569/IJACSA.2020.0110614

[31] L. Hou and Z. Li, "Fault diagnosis of rolling bearing based on tunable Q-Factor wavelet transform and convolutional neural network," International Journal of Online and Biomedical Engineering, vol. 16, no. 2, 2020, pp. 47-61. https://doi.org/10.3991/ijoe.v16i02.1 $\underline{1953}$

[32] K. Rukiye, G. Inan, C. Irem and B. Erhan. "A new method of fuzzy logic-based steganography for the security of medical images," In Proc. 23rd Signal Processing and Communications Applications Conference, SIU 2015, pp. 272-275. https://doi.org/10.1109/SIU.20 15.7129812

[33] B. Santhi and B. Dheeptha, "A novel edge-based embedding in medical images based on unique key generated using sudoku puzzle design," SpringerPlus, vol. 5, no. 1, 2016. https ://doi.org/10.1186/s40064-016-3356-1

[34] R. R. S. Tomar and K. Jain, "Lossless image compression using differential pulse code modulation and its application", In Proc. International Conference on Computational Intelligence and Communication Networks (CICN), 2015. https://doi.org/10.1109/CICN.2015. $\underline{84}$

[35] L. Ma and K. Kharasani, "Application of adaptive constructive neural networks to image compression," IEEE Transactions on Neural Networks, vol. 13, no. 5, September 2002. https://doi.org/10.1109/TNN.2002.1031943

[36] K. Sasazaki, H. Ogasawara, S. Saga, J. Maeda and Y. Suzuki, "Fuzzy vector quantization of images based on local fractal dimension," In Proc. IEEE international conference on Fuzzy Systems, 2006. https://doi.org/10.1109/FUZZY.2006.1681843

[37] K. K. Paliwal, V. Ramasubramian, "Comments on modified K-means algorithm for vector quantizer design," IEEE Transactions on Image Processing, vol 9, no 11, 2000, pp. 19641967. https://doi.org/10.1109/83.877216

[38] S. A. Durai, and E. A. Saro, "Image compression with back-propagation neural network using cumulative distribution function," International Journal of Computer, Electrical, Automation, Control and Information Engineering, vol. 2, no. 5, 2008, pp. 1571-1575. Available: https://pdfs.semanticscholar.org/ec1a/2f41ed356509b22577c79bf506203ef81d24. pdf? ga $=2.60507437 .12236357 .1596979927-99811505.1583589275$ [Accessed: June 08, 2020].

[39] B. Northan and R.D. Dony, "Image Compression with A Multiresolution Neural Network" Canadian Journal of Electrical and Computer Engineering, vol. 31, no. 1, 2006, pp. 4958. https://doi.org/10.1109/CJECE.2006.259203

\section{$7 \quad$ Author}

Abdul Khader Jilani Saudagar received his Bachelor of Engineering B. E, Master of Technology M.Tech and Doctor of Philosophy $\mathrm{PhD}$ in Computer Science \& Engineering in 2001, 2006 and 2010 respectively. His areas of interests are: Artificial Image Processing, Information Technology, Databases, Web and Mobile Application Development. He has 10 years of research and teaching experience at both undergraduate (UG) and postgraduate (PG) level. Presently working as Associate Professor 
in Information Systems Department, College of Computer \& Information Sciences (CCIS), Imam Mohammad Ibn Saud Islamic University (IMSIU), Riyadh, Saudi Arabia. He is also the Head of Intelligent Interactive Systems Research Group (IISRG) at CCIS. He was the principal investigator of the funded projects from KACST, Deanship of Scientific Research (IMSIU) and Research Development Office (RDO) Ministry of Education, Kingdom of Saudi Arabia. Dr. Saudagar has published a number of research papers in International Journals and Conferences. He is associated as member with various professional bodies like ACM, IACSIT, IAENG, ISTE etc., and working as Editorial Board member, Reviewer for many international Journals.

Article submitted 2020-07-14. Resubmitted 2020-08-05. Final acceptance 2020-08-07. Final version published as submitted by the authors. 